\title{
Comparison of Proliferative Capacity of Genetically-Engineered Pig and Human Corneal Endothelial Cells
}

\author{
Minoru Fujita ${ }^{a}$ Ruhina Mehra ${ }^{a}$ Seung Eun Lee ${ }^{a}$ Danny S. Roh ${ }^{b}$ Cassandra Long ${ }^{a}$ \\ James L. Funderburgh ${ }^{b}$ David L. Ayares ${ }^{c}$ David K.C. Cooper ${ }^{a}$ Hidetaka Hara $^{a}$ \\ ${ }^{a}$ Department of Surgery, Thomas E. Starzl Transplantation Institute ${ }^{b}$ Department of Ophthalmology, \\ Eye and Ear Institute, University of Pittsburgh, Pittsburgh, Pa., and ' Revivicor, Inc., Blacksburg, Va., USA
}

\section{Key Words}

Corneal endothelial cells • Endothelial keratoplasty •

Pig, genetically-engineered · Proliferative capacity •

Xenotransplantation

\begin{abstract}
Purpose: The possibility of providing cultured corneal endothelial cells (CECs) for clinical transplantation has gained much attention. However, the worldwide need for human (h) donor corneas far exceeds supply. The pig (p) might provide an alternative source. The aim of this study was to compare the proliferative capacity of CECs from wild-type (WT) pigs, genetically-engineered (GE) pigs, and humans. Methods: The following CECs were cultured: hCECs from donors (i) $\leq 36$ years (young), (ii) $\geq 49$ years (old), and WT pCECs from (iii) neonatal ( $<5$ days), (iv) young ( $<2$ months), and (v) old (>20 months) pigs, and CECs from young (vi) GE pigs (GTKO/ CD46 and GTKO/CD46/CD55). Proliferative capacity of CECs was assessed by direct cell counting over 15 days of culture and by BrdU assay. Cell viability during culture was assessed by annexin $V$ staining. The MTT assay assessed cell metabolic activity. Results: There was significantly lower proliferative capacity of old CECs than of young CECs $(p<0.01)$ in both pigs and humans. There was no significant difference
\end{abstract}

\section{KARGER}

Fax +4161306 1234

E-Mail karger@karger.ch

www.karger.com
(C) 2012 S. Karger AG, Basel

$0030-3747 / 13 / 0493-0127 \$ 38.00 / 0$

Accessible online at:

www.karger.com/ore in proliferative capacity/metabolic activity between young pCECs and young hCECs. However, there was a significantly higher percentage of cell death in hCECs compared to pCECs during culture $(p<0.01)$. Young GE pCECs showed similar proliferative capacity/cell viability/metabolic activity to young WT pCECs. Conclusions: Because of the greater availability of young pigs and the excellent proliferative capacity of cultured GE pCECs, GE pigs could provide a source of CECs for clinical transplantation.

Copyright $\odot 2012$ S. Karger AG, Basel

\section{Introduction}

The corneal endothelium plays an essential role in the maintenance of corneal transparency. Human (h) corneal endothelial cells (CECs) are essentially nonregenerative in vivo [1]. The loss of CECs from intraocular surgery (e.g., cataract surgery), dystrophy, or trauma triggers compensatory enlargement of the remaining CECs. Since a minimum CEC density of 500 cells $/ \mathrm{mm}^{2}$ is necessary to actively pump fluid from the stroma to the anterior chamber in the eye, loss of CECs often results in irreversible corneal endothelial dysfunction. For many years, penetrating (full-thickness) keratoplasty was the only ef- 
fective therapy to restore the CECs, but it is not without complication [2]. In recent years, new surgical procedures have been developed that replace the endothelium without corneal trephination and suturing; these include endothelial keratoplasty, e.g., posterior lamellar keratoplasty, deep lamellar endothelial keratoplasty, Descemet stripping with endothelial keratoplasty, Descemet stripping automated endothelial keratoplasty, and Descemet membrane endothelial keratoplasty [3, 4]. Irrespective of the selected keratoplasty procedure, fresh donor corneas are required to obtain the high density CECs that are necessary $\left(>2,500\right.$ cells $\left./ \mathrm{mm}^{2}\right)$ [5]. The corneal endothelium is extremely fragile and flaccid, thus difficult to transfer without damage.

Although hCECs do not have proliferative capacity in vivo and are arrested in the G1 phase of the cell cycle [6], they do have proliferative capacity in vitro [1]. The replacement of CECs with cultured CECs would provide a major advance in the treatment of corneal blindness $[3,7-9]$.

However, primary culture of hCECs is difficult, and a change in the morphology to a fibroblastic-like state cannot always be prevented. Furthermore, it is important to note that the worldwide need for human donor corneas far exceeds supply [10]. Pig (p) corneas might provide an alternative source. Corneas from specific pathogen-free pigs would avoid the potential problem of transfer of pathogenic microorganisms to the human recipient. Furthermore, stored pig corneas could be shipped to any location worldwide, and thus only a few specialized pig breeding facilities would be required. Because the cornea is an immune-privileged tissue, the evidence is that its fate as a xenograft will be significantly better than that of organ xenografts $[11,12]$.

Our recent in vitro studies have demonstrated that the human cellular and humoral immune responses to CECs from genetically-engineered (GE) pigs are no more vigorous than those to hCECs [13]. The transplantation (Tx) of cultured GE pCECs will be one potential therapeutic approach to restore the corneal endothelium. However, there have been no reports on whether the proliferative capacity of pCECs, especially GE pCECs, is similar to that of hCECs.

The aim of the present study was to compare the in vitro proliferative capacity of pCECs with that of hCECs. Furthermore, the in vitro proliferative capacity of GE pCECs was compared with that of wild-type (WT) pCECs. The present study therefore forms the basis for an innovative treatment utilizing cultured GE pCECs for Tx to correct corneal endothelial dysfunction.
Table 1. Pig corneas utilized in this study

\begin{tabular}{llll}
\hline Pig & Age & $\begin{array}{l}\text { Corneas } \\
\mathrm{n}\end{array}$ & $\begin{array}{l}\text { Mean CEC count } \\
\text { cells } / \mathrm{mm}^{2} \pm \text { SEM }\end{array}$ \\
\hline $\begin{array}{c}\text { Neonatal } \\
\text { WT }\end{array}$ & $4-5$ days & 8 & $5,959 \pm 436$ \\
$\begin{array}{c}\text { Young age } \\
\text { WT }\end{array}$ & $6-8$ weeks & 4 & $3,809 \pm 201$ \\
$\quad$ GE & & 6 & $3,768 \pm 159$ \\
$\begin{array}{c}\text { Old age } \\
\text { WT }\end{array}$ & $20-25$ months & 6 & $1,896 \pm 186$ \\
\hline
\end{tabular}

SEM = Standard error of the mean; GE = genetically-engineered: GTKO/CD46 and GTKO/CD46/CD55.

\section{Materials and Methods}

\section{Pig Corneas}

Corneas were obtained from outbred large white WT pigs (Wally Whippo, Enon Valley, Pa., USA) and from GE pigs (Revivicor, Blacksburg, Va., USA; table 1). WT pig corneas were from neonatal (4-5 days), young (6-8 weeks), and old (20-24 months) pigs (table 1). GE pig corneas were obtained from $\alpha 1,3$-galactosyltransferase gene-knockout (GTKO) pigs (that do not express the Gal $1,3 \mathrm{Gal}$ antigen that is the major target for human anti-pig antibodies) transgenic for the human complement-regulatory protein CD46 (GTKO/CD46 pigs) $[13,14]$ and from GTKO/CD46/ CD55 pigs [H. Hara, unpublished]. The density of pCECs was evaluated ex vivo using Confoscan 3 (NIDEK, Fremont, Calif., USA) [H. Hara, unpublished].

All animal care procedures were in accordance with the Principles of Laboratory Animal Care formulated by the National Society for Medical Research and the Guide for the Care and Use of Laboratory Animals prepared by the Institute of Laboratory Animal Resources and published by the National Institutes of Health.

\section{Human Corneas}

Corneas from deceased human subjects were provided by the Pittsburgh Center for Organ Recovery and Education with the approval of the University of Pittsburgh Committee for Oversight of Research Involving the Dead, and in accordance with the guidelines of the Declaration of Helsinki for research involving the use of human tissues. All human corneas (ages 18-66 years) were considered to be unsuitable for Tx because of (i) lack of a blood sample from the donor to conduct serology tests, (ii) defects of the epithelium or stroma within the optical zone, (iii) stromal infiltrates, (iv) guttata, which is one of the characteristics of corneal dystrophy, (v) an unacceptably long period $(>12 \mathrm{~h}$ ) between time of death and time of corneal excision and storage, (vi) low CEC densities, (vii) sepsis or ocular infection, or (viii) donor chemotherapy. All human corneas were stored in Optisol-GS (Bausch \& Lomb, Rochester, N.Y., USA) at $4^{\circ} \mathrm{C}$ until cell culture. Human corneas were divided into two groups (young $\leq 36$ years, old $\geq 49$ years) according to age (tables 2,3 ). 
Table 2. Details of corneas from young ( $\leq 36$ years old) human donors

\begin{tabular}{|c|c|c|c|c|c|c|}
\hline $\begin{array}{l}\text { Donor } \\
\text { No. }\end{array}$ & Age, years & Sex & Days $^{\mathrm{a}}$ & Time $^{\mathrm{b}}$ & $\begin{array}{l}\text { CEC density } \\
\text { cells } / \mathrm{mm}^{2}\end{array}$ & Cause of death \\
\hline 1 & 18 & M & 1 & $14 \mathrm{~h} 27 \mathrm{~min}$ & 2,548 & motor vehicle accident \\
\hline 2 & 19 & $\mathrm{M}$ & 2 & $17 \mathrm{~h} 55 \mathrm{~min}$ & 2,907 & toxicology \\
\hline 3 & 20 & M & 1 & $18 \mathrm{~h} 30 \mathrm{~min}$ & 3,670 & toxicology \\
\hline 4 & 20 & $\mathrm{M}$ & 6 & $14 \mathrm{~h} 20 \mathrm{~min}$ & 3,493 & asphyxia due to hanging \\
\hline 5 & 21 & $\mathrm{M}$ & 4 & $17 \mathrm{~h} 39 \mathrm{~min}$ & 3,764 & seizure disorder \\
\hline 6 & 31 & $\mathrm{M}$ & 1 & $2 \mathrm{~h} 48 \mathrm{~min}$ & 3,386 & head trauma \\
\hline 7 & 35 & $\mathrm{M}$ & 2 & $13 \mathrm{~h} 6 \mathrm{~min}$ & 3,052 & intracranial hemorrhage \\
\hline 8 & 35 & $\mathrm{M}$ & 5 & $26 \mathrm{~h} 15 \mathrm{~min}$ & 2,597 & head trauma \\
\hline 9 & 36 & M & 2 & $14 \mathrm{~h} 11 \mathrm{~min}$ & 3,434 & subdural hematoma \\
\hline Mean \pm SEM & $26.1 \pm 2.6$ & & $2.7 \pm 0.6$ & $\begin{array}{l}15 \mathrm{~h} 28 \mathrm{~min} \\
\pm 2 \mathrm{~h} 3 \mathrm{~min}\end{array}$ & $3,206 \pm 150$ & \\
\hline
\end{tabular}

${ }^{\text {a }}$ Period of time from death to culture of the corneal cells.

${ }^{b}$ Period of time from death to corneal excision and storage.

Table 3. Details of corneas from old ( $>49$ years old) human donors

\begin{tabular}{lllllll}
\hline $\begin{array}{l}\text { Donor } \\
\text { No. }\end{array}$ & Age, years & Sex & Days $^{\mathrm{a}}$ & Time $^{\mathrm{b}}$ & $\begin{array}{l}\text { CEC density } \\
\text { cells } / \mathrm{mm}^{2}\end{array}$ & Cause of death \\
\hline 1 & 47 & $\mathrm{M}$ & 1 & 12.6 & 2,420 & acute cardiac event \\
2 & 49 & $\mathrm{~F}$ & 5 & 11.26 & 3,449 & suspected drug overdose \\
3 & 49 & $\mathrm{M}$ & 3 & 13.10 & 3,014 & acute cardiac event \\
4 & 58 & $\mathrm{~F}$ & 3 & 17 & 2,321 & acute cardiac event \\
5 & 65 & $\mathrm{M}$ & 2 & 15.49 & 4,478 & acute cardiac event \\
6 & 66 & $\mathrm{~F}$ & 3 & 11.30 & 3,920 & anoxia \\
\hline Mean \pm SEM & $57.4 \pm 3.7$ & & $3.2 \pm 0.5$ & $13.9 \pm 1.8$ & $3,436 \pm 370$ & \\
\hline
\end{tabular}

a Period of time from death to culture of the corneal cells.

b Period of time from death to corneal excision and storage.

\section{Corneal Endothelial Cell Culture}

pCECs and hCECs were cultured as previously described [13]; pCECs were isolated from fresh corneas. In contrast, primary cultures of hCECs were initiated within 6 days after preservation in Optisol-GS (tables 2, 3). After primary culture, the culture medium for both pCECs and hCECs was replaced with Opti-MEM I reduced-serum medium (Invitrogen, Carlsbad, Calif., USA) containing calcium chloride $(100 \mu \mathrm{g} / \mathrm{ml}$, Sigma-Aldrich, St. Louis, Mo., USA), $0.08 \%$ chondroitin sulfate (Sigma), ascorbic acid (20 $\mu \mathrm{g} / \mathrm{ml}$, Sigma), $8 \%$ heat-inactivated fetal bovine serum (Invitrogen), antibiotic-antimycotic (Invitrogen), endothelial growth factor (30 $\mu \mathrm{g} / \mathrm{ml}, \mathrm{BD}$ Biosciences, San Diego, Calif., USA), and human epidermal growth factor $(10 \mathrm{ng} / \mathrm{ml}$, Biomedical Technologies, Stoughton, Mass., USA).

\section{Evaluation of Proliferative Capacity of CECs}

Cell Numbers. After primary culture, CECs (passage 1-3) were seeded at 5,000 cells in collagen-I-coated 12-well tissue culture plates (BD) and were cultured for 15 days. CECs were harvested and the cell number was counted every third day. The culture medium in the remaining wells was changed every third day. On each day the mean of triplicate results was expressed as a proliferation curve and compared with the rate of proliferation of each type of CEC.

Calculation of Rate of Proliferation (Doubling Time). To assess the rate of proliferation, the doubling time was calculated using the total number of CECs at 3 days and 6 days [15]. At each timepoint the results from three separate wells were averaged. The doubling time was calculated as follows:

Doubling time $=\mathrm{t} / 10(\log \mathrm{D} 2-\mathrm{D} 1)$,

where D1 is the number of CECs on day 3, D2 is the number of CECs on day 6 , and $t$ is the time interval between D1 and D2 (in this case $72 \mathrm{~h}$ ).

Cell Cycling. DNA synthesis was measured by staining of 5-bromo-2'-deoxyuridine (BrdU) using BrdU flow kit (BD). The 
60-80\% confluent cultured CECs were exposed to medium containing $10 \mu \mathrm{M} \mathrm{BrdU}$ for $30 \mathrm{~min}$. The cells were then washed to remove unbound BrdU and fixed with Cytofix/Cytoperm buffer containing $4 \%$ paraformaldehyde solution (BD) for at least 10 min. The cells were permeabilized with Cytoperm Plus Buffer (BD) for $20 \mathrm{~min}$, and incubated with DNAase (BD) for $60 \mathrm{~min}$ at $37^{\circ} \mathrm{C}$. Subsequently, the cells were incubated with 1:20 diluted FITC-conjugated anti-BrdU monoclonal antibody (BD) for 40 min at room temperature. The unbound monoclonal antibodies were washed out and cellular DNA was counterstained with 7-amino-actinomycin D (7-AAD, BD). Cellular fluorescence was measured by a LSRFortessa flow cytometer (BD).

\section{Cell Viability}

Apoptotic cells were detected by flow cytometry using annexin $\mathrm{V}$ apoptosis detection kit I (BD). CECs were harvested after $70-80 \%$ confluence, washed with PBS (Invitrogen), and resuspended with freshly prepared $1 \times$ binding buffer (BD). Then, $10^{5}$ cells per tube, including $200 \mu \mathrm{l} 1 \times$ binding buffer, were incubated with $5 \mu$ l phycoerythrin conjugated-annexin V (BD) for 30 $\mathrm{min}$ and $2.5 \mu \mathrm{l}$ 7-AAD for $20 \mathrm{~min}$ at room temperature. Annexin $\mathrm{V}$-negative/7-AAD-negative cells were identified as live cells. Annexin $\mathrm{V}$-positive/7-AAD-negative cells were identified as apoptotic, whereas annexin $\mathrm{V}$-positive/7-AAD-positive cells were identified as necrotic or dead.

\section{Metabolic Activity}

The metabolic activity of the CECs was measured with the colorimetric 3-(4,5-dimethylthiazol-2-yl)2,5-diphenyltetrazolium bromide (MTT) assay kit I (Roche Diagnostics, Indianapolis, Ind., USA). The harvested CECs were seeded in 96-well flatbottom plates (Nunc, Rochester, N.Y., USA) that had been precoated with undiluted FNC Coating Mix (Athena Enzyme Systems, Baltimore, Md., USA), at a density of 4,000, 2,000, 1,000 , and 500 cells/well. After 48 -hour culture, $10 \mu$ l MTT reagent $(5 \mathrm{mg} / \mathrm{ml})$ was added to each well and incubated for $4 \mathrm{~h}$ at $37^{\circ} \mathrm{C}$. Solubilization solution $(100 \mu \mathrm{l}$ of $10 \%$ sodium dodecyl sulfate containing $0.01 \mathrm{M} \mathrm{HCl}$ ) was added to each well, and incubated overnight in the culture incubator $\left(37^{\circ} \mathrm{C}, 5 \% \mathrm{CO}_{2}\right)$. The optical density for each sample was read with a Victor 3 Multilabel Plate Reader (PerkinElmer, Waltham, Mass., USA) at 570 $\mathrm{nm}$, with $660 \mathrm{~nm}$ used as a reference. It was necessary to correct the results because the total number of CECs in each well affected absorbance. As a parallel experiment, 4,000 CECs/well were seeded in a 96-well plate and cultured for 3 days, after which CECs were harvested and counted. Relative absorbance was calculated as follows:

Absolute absorbance $\times 4,000$ cells/absolute cell number after 3 days culture.

\section{Storage of Corneas}

To investigate the effect of storage of the cornea, excised WT pig corneas $(n=4)$ were stored in Optisol for 3-5 days before primary CEC culture. Proliferative capacity, cell viability, and metabolic activity of the CECs were compared with those of CECs from the second cornea of the same donor which had not been stored (freshly cultured).

\section{Statistical Analyses}

Data obtained from two groups were compared using the Student $t$ test, and comparisons among multiple groups were performed using the Kruskal-Wallis one-way analysis using GraphPad Prism version 4 (Graphpad Software, San Diego, Calif., USA). Values are presented as mean \pm SEM. Differences were considered to be significant at $\mathrm{p}<0.05$.

\section{Results}

\section{The Number of CECs in Pigs Decreased Significantly with Age}

The number of CECs in pigs of different ages - WT (neonatal, young, and old) and GE (young) - were counted by Confoscan 3 (table 1). The mean number of CECs from WT neonatal and young pigs was 5,959 and 3,809 cells $/ \mathrm{mm}^{2}$, respectively, whereas from old pigs the mean number was 1,895 cells $/ \mathrm{mm}^{2}$. Young GE pigs (3,767 cells/ $\mathrm{mm}^{2}$ ) showed similar CEC density to young WT pigs. The density of CECs is known to decrease significantly during life in humans $[16,17]$. As in humans, these results indicated that the number of pCECs decline with age; there was a significant correlation between the density of CECs and age $\left(\mathrm{R}^{2}=0.68, \mathrm{p}<0.01\right)$.

Human corneas were selected with at least $>2,000$ cells $/ \mathrm{mm}^{2}$ for primary culture. Mean age of young corneas was 27.4 years (mean CEC number $=3,120 / \mathrm{mm}^{2}$ ), and of old corneas 55.7 years (mean CEC number 3,267/ $\mathrm{mm}^{2}$ ) (tables 2, 3). There was no significant correlation between the number of CECs and age $\left(\mathrm{R}^{2}=0.03, \mathrm{p}=0.51\right)$.

\section{Morphology of Cultured pCECs and hCECs}

CECs from neonatal (fig. 1a) and young (fig. 1b) pigs showed typical polygonal morphology, but old pCECs had a round appearance (fig. 1c). Neonatal and young pCECs were smaller than old pCECs. Young hCECs also showed the typical homogeneous polygonal morphology (fig. 1d). In contrast, old hCEC showed larger cell size and heterogeneous morphology (fig. 1e).

\section{Young CECs Have a Significantly Greater Proliferative Capacity than Old CECs}

pCECs and hCECs of varying ages were cultured under identical conditions. Proliferation of pCECs and hCECs increased steeply after 3 days in culture, the number of CECs reaching a peak after 12-15 days (fig. 2a, b). The maximum number of pCECs and hCECs from young subjects was similar $(480,000-580,000$ cells/well or $1,200-1,500$ cells $/ \mathrm{mm}^{2}$; table 4$)$. In contrast, the maximum number of pCECs and hCECs from old subjects was 

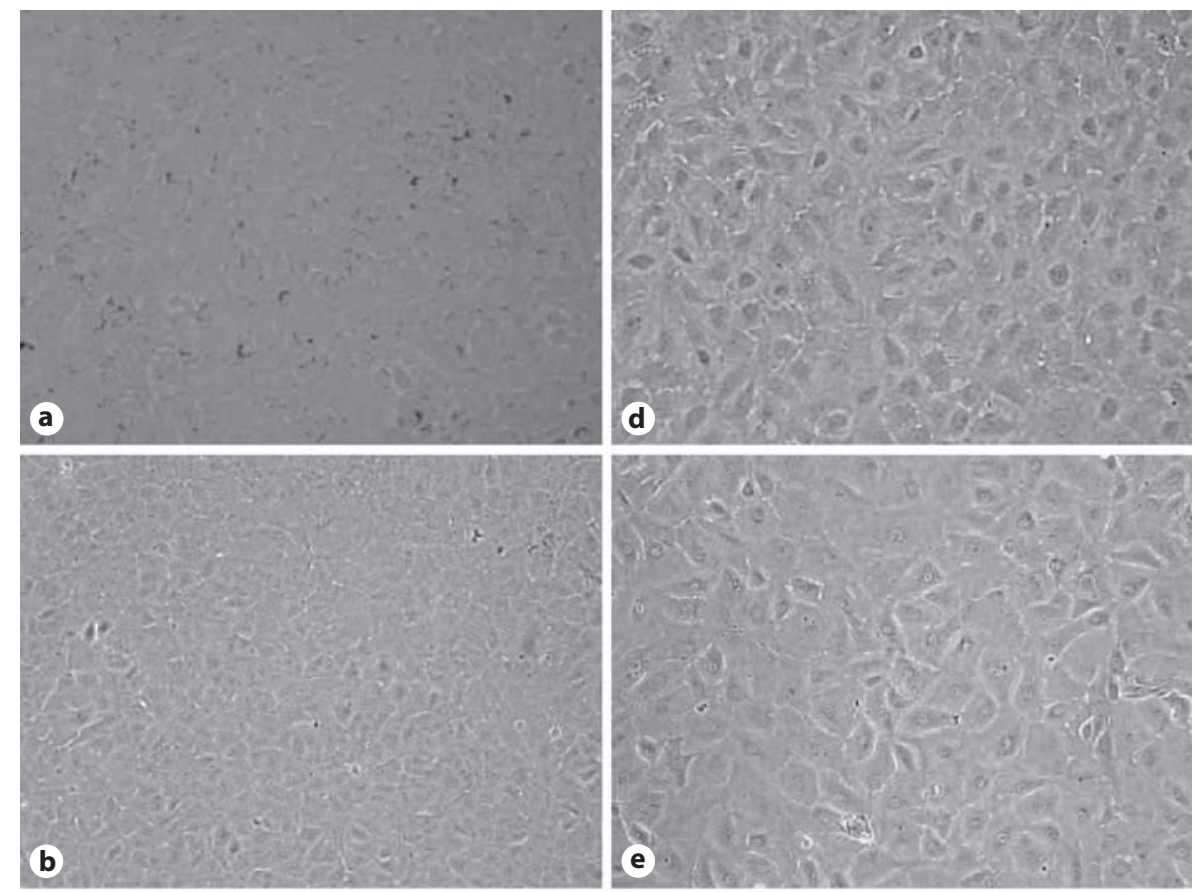

Fig. 1. Morphology of pCECs and hCECs after primary culture to confluence. $\mathrm{Mi}$ crographs of neonatal (5 days, a), young (2 months, b), and old (24 months, c) pCECs, and of young (19 years, d), and old (56 years, e) hCECs. Magnification $\times 200$.

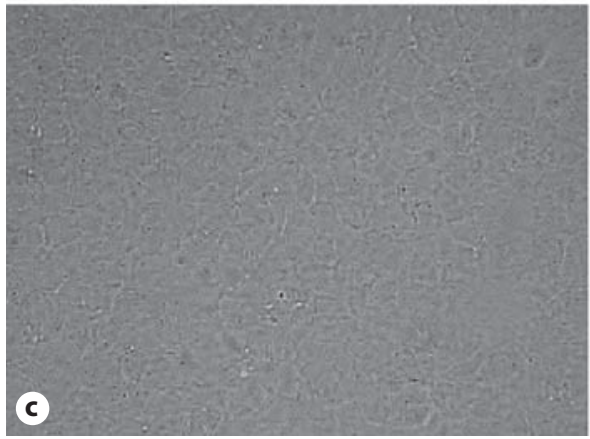

approximately $250,000-300,000$ cells/well (700-800 cells $/ \mathrm{mm}^{2}$; table 4). (It is important to note that $100 \%$ confluence of old CECs was not reached even after culture for 15 days; not shown.)

Doubling time has been measured as an estimate of cells or tumor growth in experimental and clinical situations $[18,19]$. In both pCECs and hCECs, there was a significantly longer doubling time in old than in young CECs, suggesting that young CECs have a more potent proliferative capacity (fig. 2c).

An evaluation of the activity of DNA synthesis in terms of the cell cycle provides an estimate of the proliferative capacity of cells [20]. The cell cycle status and DNA synthetic activity of cells can be determined by analyzing the correlation of total DNA and the level of incorporated BrdU by flow cytometry [21]. Staining of $\mathrm{BrdU}$ combined with 7-AAD provides the percentage of
Table 4. Maximum densities of CECs during culture

\begin{tabular}{lcll}
\hline Type of CECs & $\begin{array}{l}\text { CEC density } \\
\text { cells } / \mathrm{mm}^{2}\end{array}$ & $\begin{array}{l}\text { Versus old } \\
\text { (same species) }\end{array}$ & $\begin{array}{l}\text { Versus } \\
\text { young } \\
\text { human }\end{array}$ \\
\hline Pig & & & \\
Neonatal & $1,538 \pm 120$ & $\mathrm{p}<0.01$ & $\mathrm{NS}$ \\
Young & $1,285 \pm 113$ & $\mathrm{p}<0.05$ & $\mathrm{NS}$ \\
$\begin{array}{l}\text { Old } \\
\text { Human }\end{array}$ & $801 \pm 69$ & & $\mathrm{p}<0.05$ \\
Young & $1,474 \pm 186$ & $\mathrm{p}<0.01$ & $\mathrm{p}<0.01$ \\
Old & $702 \pm 41$ & & \\
\hline \multicolumn{2}{l}{ NS $=$ Not significant. } & & \\
a Mean \pm SEM. & & \\
\hline
\end{tabular}




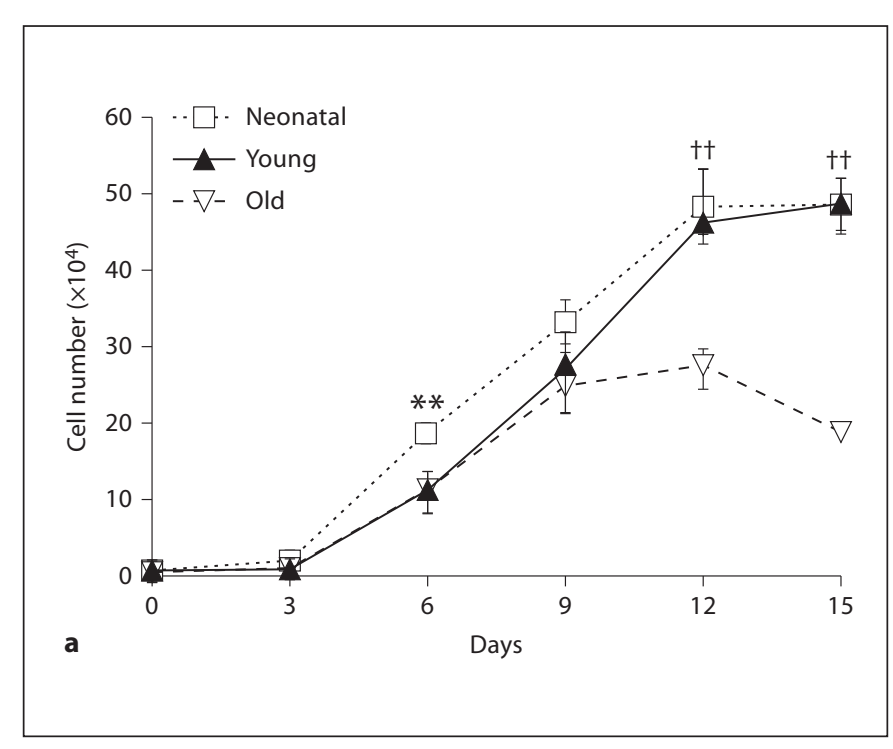

Fig. 2. Comparison of proliferative capacity between pCECs and hCECs. The proliferative capacities of neonatal, young, and old pCECs (a), and young and old hCECs (b) were compared by direct cell counting for 15 days. The maximum numbers of neonatal and young pCECs were measured after 12 days of culture, and the maximum number of old pCECs was at 9 days, which was significantly different from neonatal and young pCECs $(\mathrm{p}<0.01)$, $\left({ }^{* *} \mathrm{p}<0.01\right.$ neonatal pCECs vs. young or old pCECs, ${ }^{+\dagger} \mathrm{p}<0.01$ neonatal or young pCECs vs. old pCECs). After 6 days of culture, there were significantly fewer old hCECs than young hCECs $\left({ }^{* *} \mathrm{p}<0.01\right)$. The maximum number of young hCECs was at 9 days, in contrast to old hCECs, which was at 15 days ( $\mathrm{p}<0.01$ vs. young hCECs). $\mathbf{c}$ The doubling time of pCECs and hCECs was measured (see 'Methods'). There was a significantly more rapid doubling time of neonatal and young CECs than of old CECs in both pigs and humans $\left({ }^{* *} \mathrm{p}<0.01\right)$.

Fig. 3. Similar DNA synthesis in young pCECs and young hCEC, but less cell death in pCECs during culture. a A representative figure for cell cycle analysis using the BrdU assay by flow cytometry. Double staining with 7-AAD and BrdU demonstrated G0/G1 phase (7-AAD low and BrdU negative), $S$ phase (7-AAD positive and BrdU positive), G2/M phase (7-AAD high and BrdU negative), and dead cells (7-AAD negative and BrdU negative). b Representative figures and statistical analysis of the percentage of CECs in S phase (c) and G2/M phase (d), and dead cells (e) (showing neonatal, young, and old pCECs, and young and old hCECs).
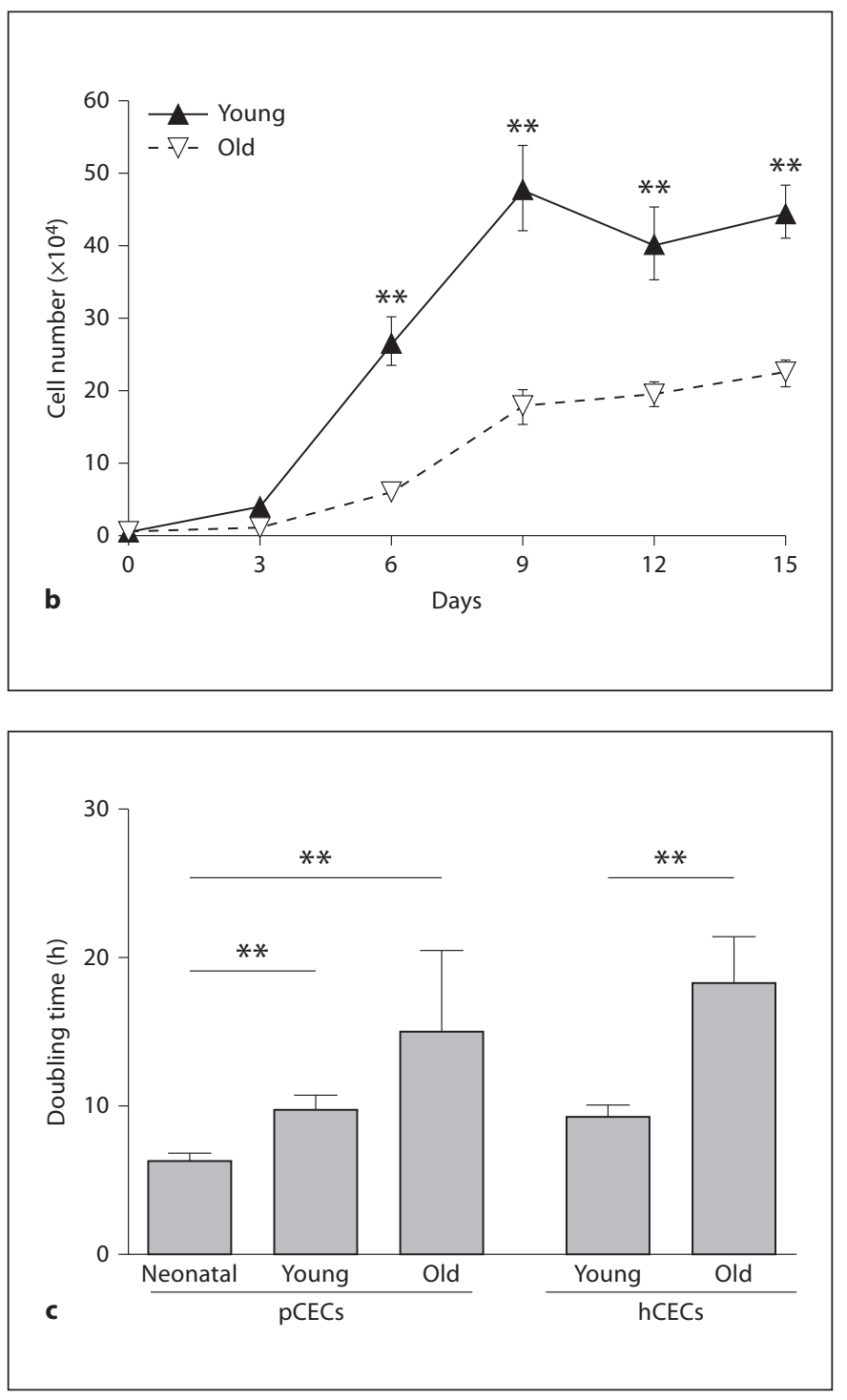

G0/G1, S, G2/M phase and dead cells were quantified by gating 7-AAD and BrdU. $c$ The percentage of cells in the $S$ phase was significantly lower in old CECs from both pigs and humans $(\mathrm{p}<$ 0.01 ). The $S$ phase of old pCECs was also significantly lower than that of young hCEC ( $<<0.01)$. d In the G2/M phase, there was no significant difference between each group of pCECs and hCECs. e During culture, there was a significantly higher incidence of cell death in hCECs compared to pCECs $(\mathrm{p}<0.01)$. There was a significantly higher percentage of dead cells in old hCECs than in young hCECs $(\mathrm{p}<0.01),\left({ }^{*} \mathrm{p}<0.05,{ }^{* *} \mathrm{p}<0.01\right)$. 


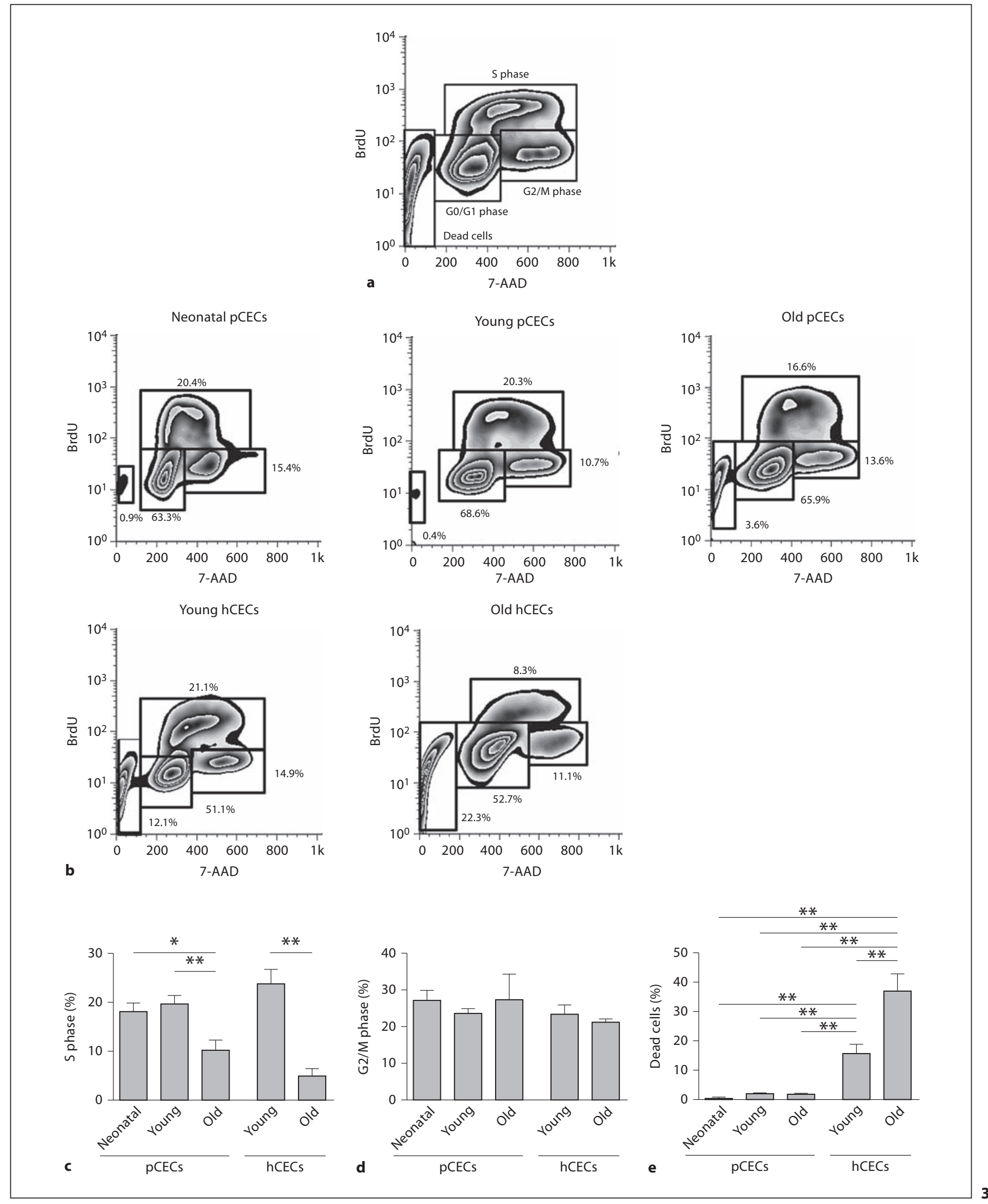


Fig. 4. During culture, there was a significantly lower incidence of apoptosis and necrosis in pCECs than in hCECs. a Representative figures of annexin $\mathrm{V}$ staining in young pCECs and hCECs. CECs were stained with annexin $\mathrm{V}$ and 7-AAD after $70-80 \%$ confluence. b During culture, there were significantly higher percentages of apoptosis and necrosis in hCECs than in pCECs, $\left({ }^{*} \mathrm{p}<0.05\right)$.
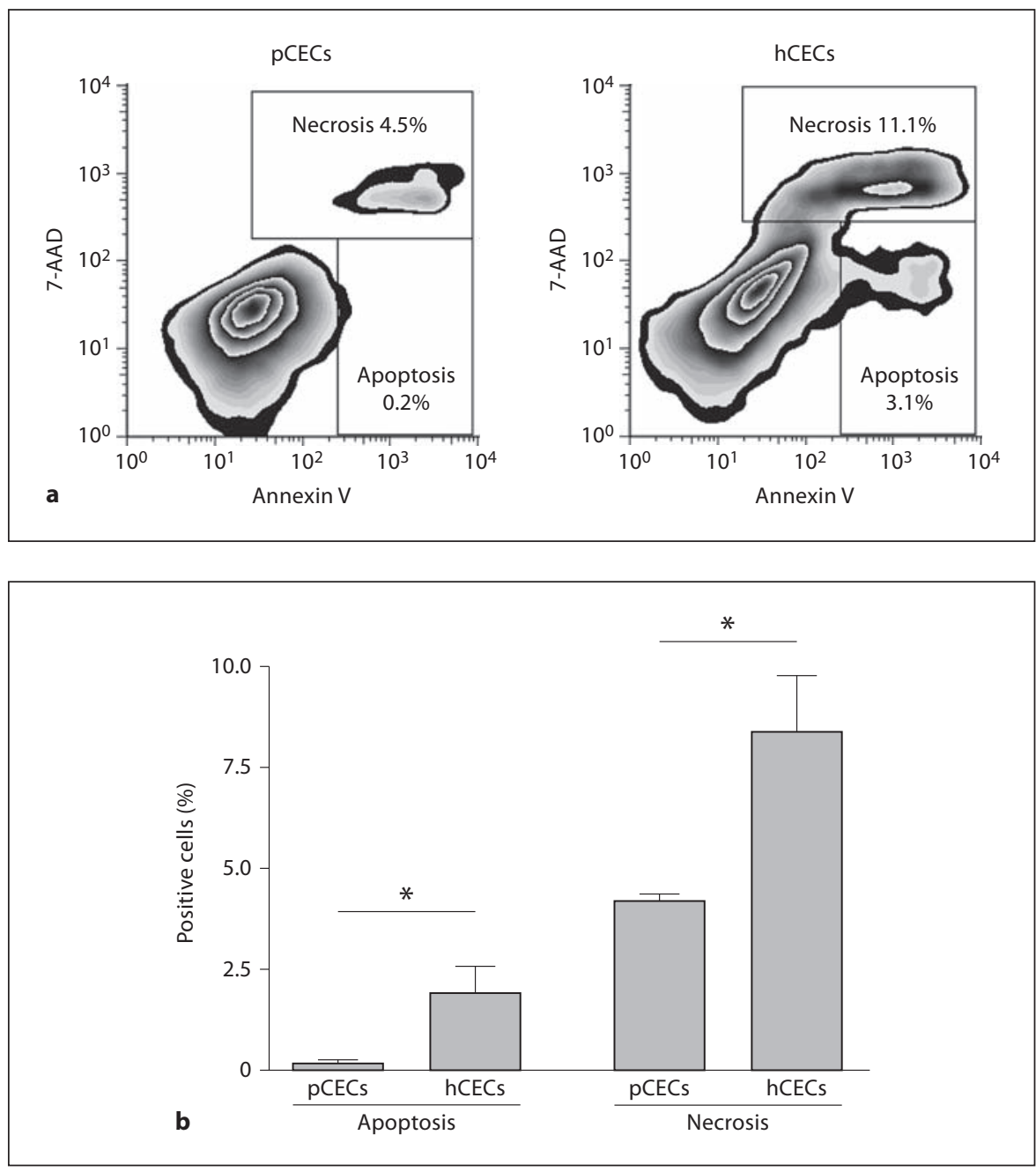

cells in a specific phase of the cell cycle, including G0/G1, $\mathrm{S}$, and G2/M phases (fig. 3a). The $S$ phase is the phase of DNA synthesis. The percentage of cells in the $S$ phase was significantly lower in old pCECs and hCECs than in young CECs ( $p<0.01$, fig. $3 \mathrm{~b}, \mathrm{c})$. These results correlated with cell counts (fig. 1). In contrast to the $S$ phase, there was no significant difference in the G2/M phase between pCECs and hCECs of different ages (fig. 3d).

These results indicated that the proliferative capacity of neonatal and young pCECs was similar to that of young hCECs. However, both young and old hCECs showed a significantly higher incidence of cell death during culture compared to pCECs $(\mathrm{p}<0.01$; fig. $3 \mathrm{~b}$, e). In addition, old hCECs showed a higher incidence of cell death during culture compared to young hCECs ( $\mathrm{p}<$ 0.01 ; fig. 3e). There was no significant difference in the incidence of cell death during culture among pCECs of different ages.

\section{Less Viability of hCECs during Culture Compared to pCECs}

To further confirm whether hCECs showed a greater susceptibility to cell death during culture, the number of apoptotic cells was investigated. The incidence of both apoptosis (annexin V-positive/7-AAD-negative) and necrosis (annexin V-positive/7-AAD-positive) in hCECs was significantly greater than in pCECs (fig. 4a, b).

\section{Similar Metabolic Activity between Pig and Human CECs}

To investigate whether the reduced proliferative capacity of old CECs was associated with less metabolic ac- 

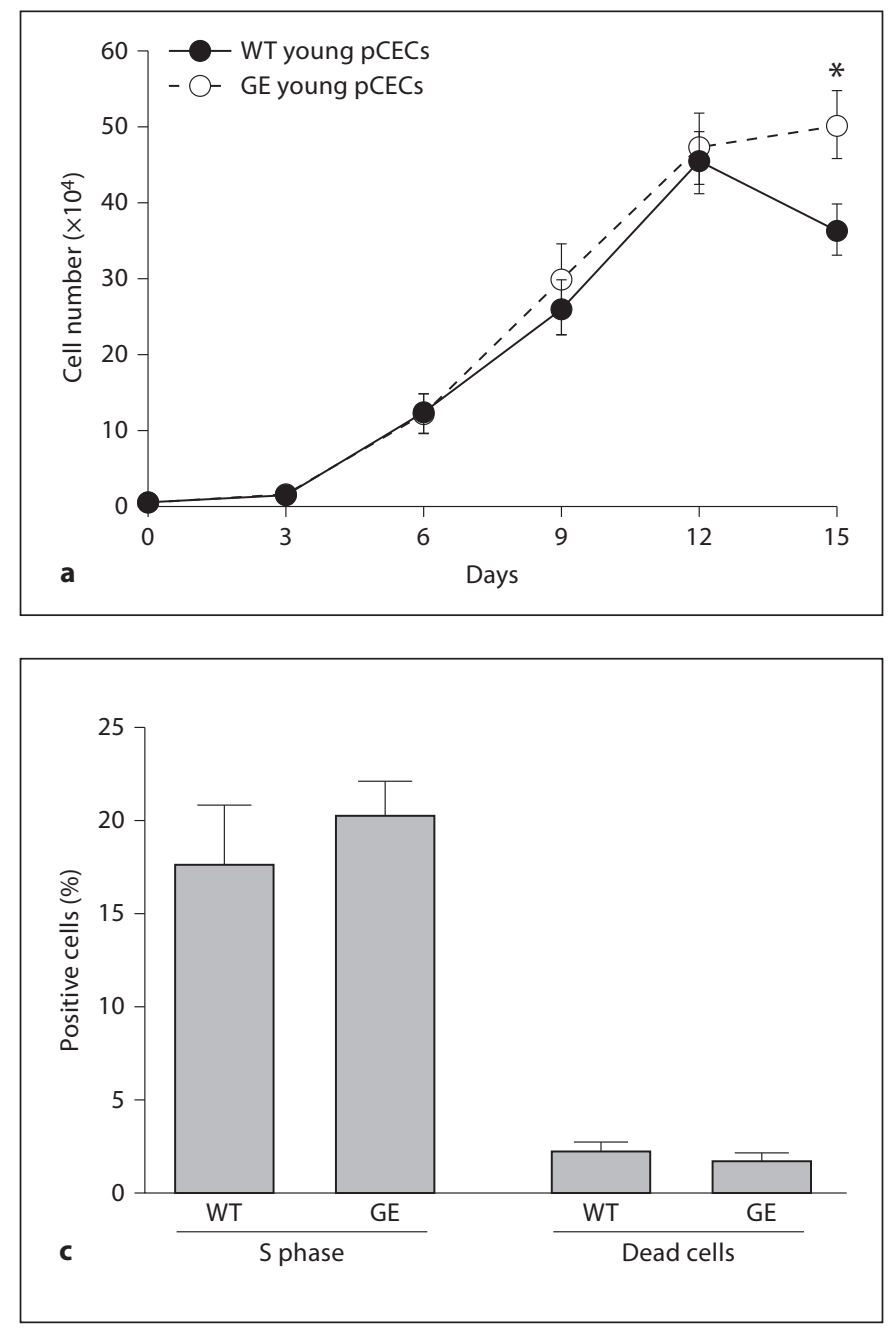

Fig. 5. GE pCECs have similar characteristics to WT pCECs. Proliferation curve (a), doubling time (b), DNA synthesis (S phase) and cell viability (c), and metabolic activity (d) were compared between young WT $(\mathrm{n}=4)$ and young GE $(\mathrm{n}=6)$ pCECs. a Proliferation capacities of young WT and young GE pCECs were similar, except at 15 days, $\left({ }^{*} \mathrm{p}<0.05\right)$. b There was no difference in dou-

tivity, the MTT assay was carried out after 3 days of culture. Since proliferation of both pCECs and hCECs increased steeply after 3 days in culture, measurement at 3 days provided an estimate of metabolic activity before proliferation increased. There was no significant difference in metabolic activity between pCECs and hCECs, or between young and old CECs (data not shown), suggesting that, during the early period of culture, any differences in proliferative capacity of CECs were not related to metabolic activity.

Pig Corneal Endothelial Cell Culture
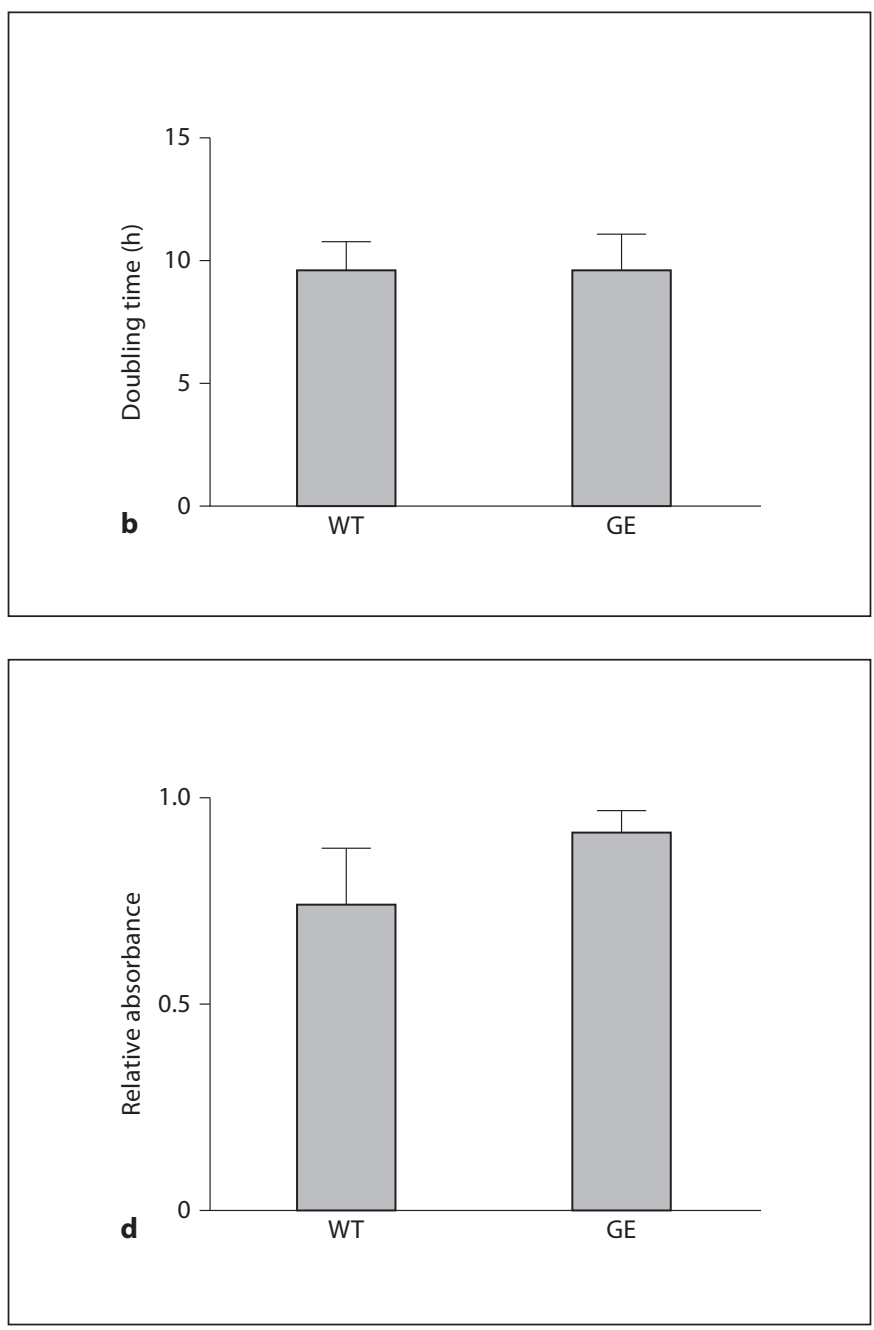

bling time between young WT and GE pCECs. c The BrdU assay demonstrated no difference in the percentage of cells in the $S$ phase and in the number of dead cells between WT and GE pCECs. $\mathbf{d}$ There was no difference in metabolic activity between WT and GE pCECs.

GE pCECs Had Similar Proliferative Capacity, Cell Viability, and Metabolic Activity as WT pCECs

There was no significant difference in cell numbers (fig. 5a), doubling time (fig. 5b), DNA synthesis, cell viability (fig. 5c), of metabolic activity (fig. 5d) between GE and WT pCECs.

\section{Storage of CECs Had No Effect on Proliferative}

Capacity, Cell Viability, or Metabolic Activity

Human corneas had been stored in Optisol until primary culture, and this may have affected proliferative ca- 
pacity and cell viability. To investigate this possibility, freshly cultured young pCECs were compared to stored young pCECs. There was no significant difference in proliferative capacity, cell viability, or metabolic activity between stored and fresh pCECs (data not shown).

\section{Discussion}

Corneal Tx has advanced rapidly during the past decade, with a shift in paradigm from full-thickness penetrating keratoplasty to partial-thickness (component) corneal Tx. Such partial-thickness Tx replaces the diseased tissue, but not the entire cornea. In particular, endothelial keratoplasty has received more attention in the clinic because it has clear advantages over penetrating keratoplasty, such as faster recovery of visual acuity, increased tectonic strength, and lower risk of intraoperative complications [22]. However, the availability of human corneas for endothelial keratoplasty is reduced by (i) a long delay after donor death before excision of the corneas, or (ii) technical difficulties because CEC damage has occurred during the handling of these fragile donor corneas. Even under the best conditions for Tx, subsequent graft failure from immunologic rejection or nonimmunologic endothelial decompensation may still occur [5].

Recently, the in vitro culture of hCECs has been considered as an alternative method for replacing damaged corneal endothelium [9]. Clinical observations suggest that transfer of CECs into the recipient anterior chamber might be sufficient to restore corneal transparency through endothelial cell migration [23]. The concept of using a carrier to implant ex vivo cultured CECs into the recipient anterior chamber may become an increasingly realistic approach $[8,24-26]$. Hence, the ability to culture hCECs in vitro with relative consistency has stimulated research into the development of a suitable delivery system in the form of a synthetic or biological carrier. Tx of cultured corneal endothelium has been attempted in animal models to establish a new intervention for corneal endothelial dysfunction $[8,26]$.

In addition, a Rho-associated kinase inhibitor promotes cultured monkey CEC proliferation both in vitro and in vivo $[27,28]$. However, there are still concerns regarding the use of human corneas to produce cultured CECs for Tx. These include difficulties in hCEC culture, the questionable proliferative capacity of old hCECs, immunological rejection and/or nonimmunological CEC damage, particularly in high-risk patients (highly sensitized to donor HLA antigens, or with an inflamed cor- neal bed), and the worldwide shortage of suitable human corneas.

Pigs could provide an alternative source of CECs for Tx. The use of CECs from GE pigs offers resistance to the human immune responses $[10,11,13]$. As with hCECs, pCECs cannot proliferate in vivo [29], but can do so in vitro [13]. However, it was important to investigate whether pCECs have a similar or greater proliferative capacity in vitro than hCECs, and to determine whether old pigs can be used as sources for cultured CECs for Tx. The proliferative capacity of young ( $<39$ years) hCECs was greater than that of old ( $>49$ years) hCECs. This observation has also been reported by others [30], and indicates that young hCECs are more suitable for CEC Tx than old hCECs.

We used neonatal CECs (5-7 days) and young (6-8 weeks) and old (20-24 months) pigs for our study. Young pCECs demonstrated greater proliferative capacity than old pCECs. Although old pigs ( $>20$ months) are approximately equivalent in age to a 25 -year-old human, old pCECs showed significantly less proliferative capacity than young hCECs ( $<39$ years), suggesting that hCECs may have a greater proliferative capacity at the equivalent age. We could not compare young pCECs ( $<2$ months), which are approximately equivalent to a 3-year-old human, because of the unavailability of human corneas from young children. However, young pCECs showed a similar proliferative capacity to hCECs from young adults ( $<36$ years).

These results indicated that human and pig corneas from young subjects might be an ideal source for in vitro expansion of CECs to provide cultured corneal endothelial sheets. However, young human corneas are difficult to obtain because the majority of high-quality corneas from young deceased humans are used in the clinic for corneal Tx. Corneas from healthy young donors are fewer than those from older donors, and, furthermore, most human corneas that become available for CEC culture are considered unsuitable for clinical corneal Tx (e.g., because of a prolonged period between donor death and corneal excision and storage, or low CEC density) and therefore will be of poorer quality.

In addition, there was significantly less cell death during culture in pCECs compared to hCECs. In particular, a higher percentage of cell death was found in old hCECs compared to young hCECs. This may contribute to difficulty in culturing hCECs. In addition, susceptibility to cell death in hCECs during culture might lead to an increased incidence of CEC loss after Tx from immunological and/or nonimmunological mechanisms.

Although collagen type I has been widely used for cell culture, including the culture of human vascular endo- 
thelial cells [14], it may not be ideal for the culture of hCECs. However, if the cells are already being grown in a 'coated' plate or flask to support cell attachment, we believe the most important factor for cell culture is the medium. Plates with different 'coatings' have been used, such as chondroitin sulfate [31], FNC Coating Mix [19], and collagen type IV [32]. A preliminary study in our laboratory indicated no significant difference in CEC proliferation capacity and morphology during culture in collagen type I- or FNC-coated plates (data not shown). In addition, a recent report suggests that matrix-coating may not be mandatory for the culture of hCECs [33]. Therefore, coating plates with collagen type I would appear to have little effect on CEC proliferation capacity.

We have previously demonstrated the significant expression of Gal $\alpha 1,3 \mathrm{Gal}$ on cultured WT pCECs, and upregulation of this antigen when cells were activated, which resulted in increased complement-mediated cytotoxicity by pooled human sera [13]. It would therefore be beneficial, if not essential, to use CECs from GTKO pigs to reduce the human humoral immune response. We demonstrated that human humoral and cellular immune responses to GTKO/CD46 pCECs were significantly reduced compared to WT pCECs [13]. GTKO/CD46/CD55 pigs have now been produced by Revivicor, and CECs from these pigs are resistant to complement-dependent lysis by pooled human sera [Hara et al., unpublished]. The hCD4 ${ }^{+}$ T cell responses to nonactivated GTKO/CD46 pCECs are similar to those to hCECs, although they are increased when CECs are activated. The hCD4 ${ }^{+} \mathrm{T}$ cell response to activated GTKO/CD46 pCECs is significantly greater than to hCECs. Pigs transgenic for class II transactivator knock-down [34] and pCTLA4-Ig transgenic pigs [35, 36] may reduce the $\mathrm{hCD} 4^{+} \mathrm{T}$ cell immune response.

Further studies are required before cultured GE pCECs could be transplanted successfully into primates. These include (i) the phenotypic character of the cultured CECs after several passages, (ii) the expression pattern of function-related proteins, e.g., $\mathrm{ZO}-1$ and $\mathrm{Na}+\mathrm{K}+-$ ATPase, and (iii) functional tests of cultured CECs using in vivo corneal endothelial dysfunction models [37].

In conclusion, the clinical application of cultured pCECs for Tx would provide a new approach to the treatment of corneal endothelial dysfunction. Our studies suggest the feasibility of providing corneal endothelial sheets derived from young GE pCECs for clinical Tx because (i) young pCECs have a similar proliferative capacity to young hCECs, (ii) pCECs show less cell death during culture compared to hCECs, and thus we believe are easier to culture compared to hCECs, (iii) there is a greater availability of corneas from young pigs than young humans, (iv) GE pCECs are more resistant to the human immune response, and (v) a single GE pig cornea could provide enough cultured CECs for several patients. Optimistically, we believe that GE pigs will open new avenues by which researchers can use cultured GE pCECs to treat corneal blindness.

\section{Acknowledgments}

We thank Janice C. Anderson, Theodore Castellanos, and Karen Brown from the Pittsburgh Center for Organ Recovery and Education (CORE) for providing human corneas for these studies. Minoru Fujita, MD, PhD, is the recipient of Kawasaki Sukenobu Memorial Fund of Kawasaki Medical School for Research Study, Japan, and Kawasaki Medical School Alumini Association Fund for Foreign Study, Japan.

This work was supported in part by NIH grants U01 (AI068642, D.K.C.C), R21 (A1074844, D.K.C.C.) and RO3 (AI096296, H.H.), by Olympus America Inc. (No. 2115, H.H.), and by Sponsored Research Agreements between the University of Pittsburgh and Revivicor, Inc., Blacksburg, Va., USA.

\section{Disclosure Statement}

D.L.A. is an employee of Revivicor, Inc., and owns stock in the company.

\section{References}

1 Joyce NC: Proliferative capacity of the corneal endothelium. Prog Retin Eye Res 2003; 22:359-389.

2 Glasser DB: Changing trends in keratoplasty. Am J Ophthalmol 2011;151:394-396.

3 Engelmann K, Bednarz J, Valtink M: Prospects for endothelial transplantation. Exp Eye Res 2004;78:573-578.

4 Dapena I, Ham L, Melles GR: Endothelial keratoplasty: DSEK/DSAEK or DMEK - the thinner the better? Curr Opin Ophthalmol 2009;20:299-307.
5 Terry MA, Shamie N, Chen ES, Hoar KL, Phillips PM, Friend DJ: Endothelial keratoplasty: the influence of preoperative donor endothelial cell densities on dislocation, primary graft failure, and 1-year cell counts. Cornea 2008;27:1131-1137.

6 Joyce NC, Meklir B, Joyce SJ, Zieske JD: Cell cycle protein expression and proliferative status in human corneal cells. Invest Ophthalmol Vis Sci 1996;37:645-655.
7 Sumide T, Nishida K, Yamato M, Ide T, Hayashida Y, Watanabe K, Yang J, Kohno C, Kikuchi A, Maeda N, Watanabe H, Okano T, Tano Y: Functional human corneal endothelial cell sheets harvested from temperature-responsive culture surfaces. FASEB J 2006;20:392-394.

8 Koizumi N, Sakamoto Y, Okumura N, Okahara N, Tsuchiya H, Torii R, Cooper LJ, Ban Y, Tanioka H, Kinoshita S: Cultivated corneal endothelial cell sheet transplantation in a primate model. Invest Ophthalmol Vis Sci 2007;48:4519-4526. 
9 Peh GS, Beuerman RW, Colman A, Tan DT, Mehta JS: Human corneal endothelial cell expansion for corneal endothelium transplantation: an overview. Transplantation 2011;91:811-819.

10 Hara H, Cooper DK: Xenotransplantation the future of corneal transplantation? Cornea 2011;30:371-378.

-11 Hara H, Cooper DK: The immunology of corneal xenotransplantation: a review of the literature. Xenotransplantation 2010;17: 338-349.

12 Kim MK, Wee WR, Park CG, Kim SJ: Xenocorneal transplantation. Curr Opin Organ Transplant 2011;16:231-236.

-13 Hara H, Koike N, Long C, Piluek J, Roh DS, Sundarraj N, Funderburgh JL, Mizuguchi Y, Isse K, Phelps CJ, Ball SF, Ayares DL, Cooper DK: Initial in vitro investigation of the human immune response to corneal cells from genetically-engineered pigs. Invest Ophthalmol Vis Sci 2011;52:5278-5286.

14 Hara H, Long C, Lin YJ, Tai HC, Ezzelarab $\mathrm{M}$, Ayares D, Cooper DK: In vitro investigation of pig cells for resistance to human antibody-mediated rejection. Transpl Int 2008; 21:1163-1174.

15 Shimizu K, Koga H, Takeda M, Mikami Y, Tsunoda T, Iida M, Haruma K: Suddenly accelerated growth of gastrointestinal stromal tumor of remnant stomach. Dig Dis Sci 2003 48:1106-1109.

16 Nucci P, Brancato R, Mets MB, Shevell SK: Normal endothelial cell density range in childhood. Arch Ophthalmol 1990;108:247248.

-17 Bourne WM, Nelson LR, Hodge DO: Central corneal endothelial cell changes over a tenyear period. Invest Ophthalmol Vis Sci 1997; 38:779-782.

18 Baserga R: The relationship of the cell cycle to tumor growth and control of cell division: a review. Cancer Res 1965;25:581-595.

19 Konomi K, Zhu C, Harris D, Joyce NC: Comparison of the proliferative capacity of human corneal endothelial cells from the central and peripheral areas. Invest Ophthalmol Vis Sci 2005;46:4086-4091.
20 Chen J, Guerriero E, Lathrop K, SundarRaj $\mathrm{N}$ : Rho/ROCK signaling in regulation of corneal epithelial cell cycle progression. Invest Ophthalmol Vis Sci 2008;49:175-183.

-21 Dolbeare F, Gratzner H, Pallavicini MG, Gray JW: Flow cytometric measurement of total DNA content and incorporated bromodeoxyuridine. Proc Natl Acad Sci USA 1983; 80:5573-5577.

22 Lee WB, Jacobs DS, Musch DC, Kaufman SC, Reinhart WJ, Shtein RM: Descemet's stripping endothelial keratoplasty: safety and outcomes: a report by the American Academy of Ophthalmology. Ophthalmology 2009;116:1818-1830.

23 Balachandran C, Ham L, Verschoor CA, Ong TS, van der Wees J, Melles GR: Spontaneous corneal clearance despite graft detachment in Descemet membrane endothelial keratoplasty. Am J Ophthalmol 2009; 148:227-234, e221.

-24 Mimura T, Yamagami S, Yokoo S, Usui T, Tanaka K, Hattori S, Irie S, Miyata K, Araie M, Amano S: Cultured human corneal endothelial cell transplantation with a collagen sheet in a rabbit model. Invest Ophthalmol Vis Sci 2004;45:2992-2997.

25 Lai JY, Chen KH, Hsiue GH: Tissue-engineered human corneal endothelial cell sheet transplantation in a rabbit model using functional biomaterials. Transplantation 2007; 84:1222-1232.

26 Koizumi N, Sakamoto Y, Okumura N, Tsuchiya H, Torii R, Cooper LJ, Ban Y, Tanioka H, Kinoshita S: Cultivated corneal endothelial transplantation in a primate: possible future clinical application in corneal endothelial regenerative medicine. Cornea 2008;27(suppl 1):S48-S55.

27 Okumura N, Koizumi N, Ueno M, Sakamoto Y, Takahashi H, Hirata K, Torii R, Hamuro J, Kinoshita S: Enhancement of corneal endothelium wound healing by Rho-associated kinase (ROCK) inhibitor eye drops. Br J Ophthalmol 2011;95:1006-1009.

28 Okumura N, Ueno M, Koizumi N, Sakamoto Y, Hirata K, Hamuro J, Kinoshita S: Enhancement on primate corneal endothelial cell survival in vitro by a ROCK inhibitor. Invest Ophthalmol Vis Sci 2009;50:36803687.
29 Singh G, Bohnke M, Draeger J: Endothelial wound healing in organ-cultured pig corneae after mechanical trauma. Fortschr Ophthalmol 1984;81:293-295.

30 Zhu C, Joyce NC: Proliferative response of corneal endothelial cells from young and older donors. Invest Ophthalmol Vis Sci 2004;45:1743-1751.

31 Engelmann K, Friedl P: Growth of human corneal endothelial cells in a serum-reduced medium. Cornea 1995;14:62-70.

32 Ishino Y, Sano Y, Nakamura T, Connon CJ, Rigby H, Fullwood NJ, Kinoshita S: Amniotic membrane as a carrier for cultivated human corneal endothelial cell transplantation. Invest Ophthalmol Vis Sci 2004;45: 800-806

33 Li W, Sabater AL, Chen YT, Hayashida Y, Chen SY, He H, Tseng SC: A novel method of isolation, preservation, and expansion of human corneal endothelial cells. Invest Ophthalmol Vis Sci 2007;48:614-620.

34 Hara H, Crossley T, Witt W, Long C, Fang J, Ezzelarab M, Starzl TE, Ayares D, Cooper DK, Dai Y: Dominant-negative CIITA transgenic pigs - effect on the human anti-pig $\mathrm{T}$ cell immune response and immune status. Am J Transplant 2010;10:187.

35 Phelps CJ, Ball SF, Vaught TD, Vance AM, Mendicino M, Monahan JA, Walters AH, Wells KD, Dandro AS, Ramsoondar JJ, Cooper DK, Ayares DL: Production and characterization of transgenic pigs expressing porcine CTLA4-Ig. Xenotransplantation 2009; 16:477-485.

-36 Koshika T, Phelps C, Fang J, Lee SE, Fujita M Ayares D, Cooper DK, Hara H: Relative efficiency of porcine and human cytotoxic Tlymphocyte antigen 4 immunoglobulin in inhibiting human CD4(+) T-cell responses co-stimulated by porcine and human B7 molecules. Immunology 2011;134:386-397.

37 Koizumi N, Okumura N, Kinoshita S: Development of new therapeutic modalities for corneal endothelial disease focused on the proliferation of corneal endothelial cells using animal models. Exp Eye Res 2012;95:6067. 